\title{
QoS-Enabled Service Discovery Using Agent Platform*
}

\author{
Kee-Hyun Choi, Ho-Jin Shin, and Dong-Ryeol Shin \\ School of Information \& Communication Engineering Sungkyunkwan University, Korea \\ \{gyunee, hjshin, drshin\}@ece.skku.ac.kr \\ http://nova.skku.ac.kr
}

\begin{abstract}
As the number of Internet services is growing rapidly, the network users need a framework to locate and utilize those services that are available in the Internet. As a result, dynamic service discovery plays an important role in certain networks, such as P2P networks. Recently many P2P mechanisms have been proposed which focus on the naming and discovery protocols. However, because these mechanisms do not provide congestion management and prioritybased scheduling in P2P networks, they cannot provide an efficient communication among the nodes. Based on these observations, in this paper, we propose a QoS-based agent framework, designed to resolve such problems, and demonstrate its effectiveness.
\end{abstract}

\section{Introduction}

Service discovery is increasing in importance as its usage becomes more and more widespread throughout the Internet. The entire premise of the Internet is centered on the sharing of information and services. The focus of P2P networks is the mechanism by which a peer provides other peers with services and discovers the services availabe from other peers. In such an environment, a peer can be any network-aware device such as cell phones, PDA, PC or any device you can imagine that passes information in and out. In some cases, a peer might be an application distributed over several machines.

A large number of papers dealing with service discovery have recently been published. Although the schemes proposed provide efficient and fast searching mechanisms for service discovery, they do not consider QoS. With these observations in mind, we developed a new agent platform designed to provide QoS and evaluated its effectiveness by simulation, which is a main contribution of this paper. The remainder of this paper is organized as follows. Section 2 describes the proposed agent platform. In section 3, we present the simulation results for the proposed system. Finally, we conclude this paper in Section 4.

\section{Proposed Agent Platforms}

In this section, we describe our proposed Agent Platform (AP) architecture. The overall design of the architecture is shown in Figure 1. We adopt the FIPA[1] reference

\footnotetext{
* This research was partially supported by a grant from the CUCN, Korea and Korea Science \& Engineering Foundation (R01-2004-000-10755-0).
} 


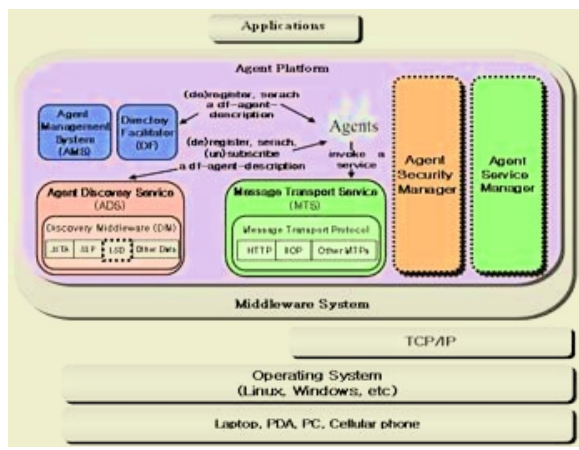

Fig. 1. Agent Platform
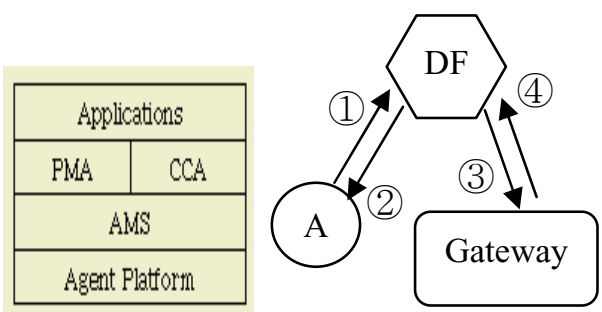

Fig. 2. a) Agent platform b) Setup sequence

model and add new components. The Agent Service Manager is a QoS solution for ad-hoc networks and P2P networks. By using the ASM, we allow our agent platform to provide QoS in P2P networks. The ASM uses two separated but not independent management agents. The PMA provides dynamic priority information according to the network status. When an application wants to transmit its data, it contacts the DF, in order to obtain priority information from the PMA. In this way, each application can control its traffic according to the priority assigned by the PMA. The DF updates the priority information whenever the network status changes. Figure 2-b) shows the setup procedure. Table 1 shows the priority level of each type of service. The DF classifies the traffic according to the priority information. When the DF receives a service request message, it returns the current priority information. We can control congestion by using the second special agent, which is called the CCA. The CCA also uses priority information, just as the PMA does. However, the CCA verifies the priority information by periodically sending a check messages. Thus, applications (e.g., $\mathrm{ftp}$, telnet, multimedia, etc.) can adjust their sending rate accordingly. By means of these two agents, we can adjust the data rate at the local system (e.g., PC, PDA, and Notebook, etc) whenever the network traffic is changed. Moreover, the network traffic can be classified using the information in the gateway. Thus, the priority is not fixed but adjustable according to the network environment.

\section{Simulations}

This section presents the performance results of the priority-based service discovery (PSD) mechanism. We simulate our mechanism with/without an infrastructure (e.g., AP, gateway).

\subsection{Simulation with Infrastructure}

We simulate these mechanisms using a simple dumbbell network model in a wired network. The gateway has ten traffic sources and the nodes, which are attached to the gateway, are wireless nodes. These wireless nodes do not move in our simulation. In Link2, we test both the droptail queuing mechanism and the proposed priority-based 


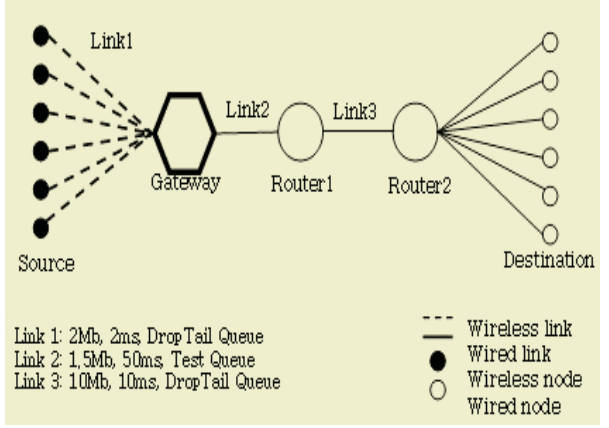

Fig. 3. Network Topology
Table 1. Priority Level

\begin{tabular}{|c|c|}
\hline Services & priority \\
\hline Service advertisements & 1 \\
\hline Service requests & 2 \\
\hline Event messages & 3 \\
\hline Voice traffic & 4 \\
\hline Multimedia & 5 \\
\hline E-commerce & 6 \\
\hline Light data traffic & 7 \\
\hline Bulk data traffic & 8 \\
\hline
\end{tabular}

queuing mechanism. Figure 3 depicts the network topology. Using the NS simulator [2], a simulation was conducted in order to determine to what extent the proposed scheme improves the time-limited traffic as compared to the conventional mechanisms (droptail queue in this paper). We use TCP traffics for both the light and bulk data traffic. Each TCP flow uses an FTP application. We change the maximum congestion window size of each TCP flow according to its priority. High priority traffic is either CBR or EXP traffic. Figure 4 shows the simulation results. Even if the number of traffic sources increases, the CBR traffic using PSD does not slow down, whereas the CBR traffic with the droptail queuing mechanism does. Moreover, the TCP traffic with PSD shows the almost the same result as that with the droptail mechanism, which implies that the proposed scheme improves the performance of time-limited traffic without affecting the TCP traffic.

\subsection{Simulation in Ad-Hoc Network}

We modify the DFS[3] mechanism using the priority information. The DFS protocol for WLANs allocates bandwidth to flows in proportion to their weights and accounts for variable packet sizes. The DFS schedules packets for transmission based on their eligibility. Because of the distributed nature of the DFS protocol, collisions may occur, which causes priority reversal and undermine the fairness. Although DFS provides fairness and better throughput, the backoff interval is directly proportional to the Packet-length/Weight ratio in the linear scheme of DFS. For this reason, we use the priority-based backoff algorithm in enhanced DFS[4]. The scenario and parameters used in this paper are identical to those used in DFS. We perform simulations based on the implementation described in [3]. The channel bandwidth is set to $2 \mathrm{Mbps}$. The simulation environment consists of $\mathrm{n}$ nodes. All of the nodes are stationary and are in transmission range of each other, in order to simulate a broadcast LAN. The number of nodes is always even. In this paper, identical flows refer to flows that are always backlogged and have equal packet size. Figure 5 shows the throughput results for the PSD, DFS and DCF mechanisms. We note that PSD not only guarantees fairness but also improves the throughput of time-limited traffics. 


\section{Conclusions}

With the increasing number of people using mobile devices such as PDAs, cellphones, notebooks, etc., the need to connect them to the Internet for ubiquitous sharing and data access is increasing. An efficient service discovery infrastructure plays an important role in such a dynamic environment. We reviewed a number of existing service discovery frameworks and found that they all suffer from one common problem, namely, a lack of QoS-based service discovery. In this paper, we introduced an agent platform designed to support QoS management. The simulation results show that the proposed mechanism improves the performance of time-limited traffic, without affecting that of other traffic, and guarantees fairness in an ad-hoc network.

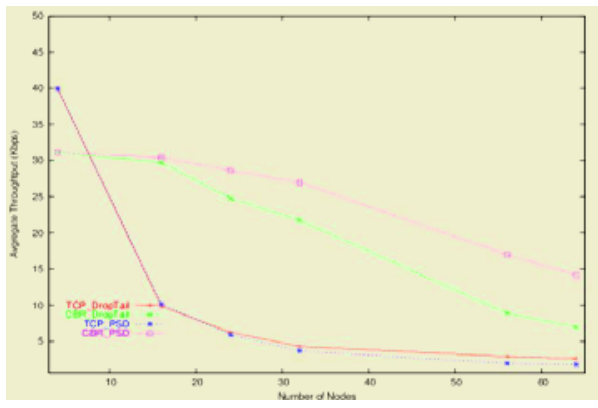

Fig. 4. Aggregated throughput in wired network

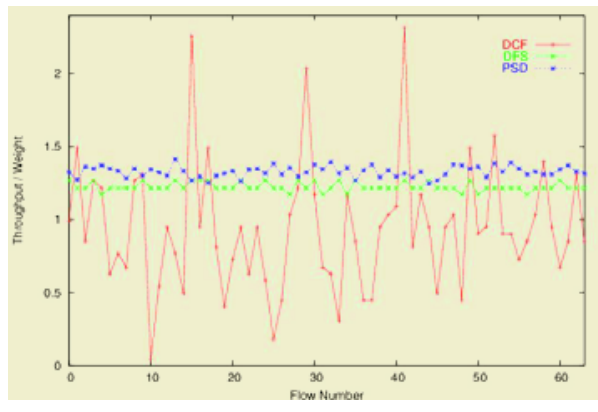

Fig. 5. Throughput of 64 flows in wireless network

\section{References}

1. FIPA: Agent Management Specification, Dec. 2002.

2. K. Fall and K. Varadhan, "ns Notes and documentation", tech. rep., VINT Project, UCBerkeley and LBNL(2003)

3. N. H. Vaidya, P. Bahl, and S. Gupta, "Distributed Fair Scheduling in a Wireless LAN", In Proc. Of ACM MOBICOM2000, Boston, MA USA(200) 167-178

4. K.H Choi, H.J. Shin and Dong-Ryeol Shin, "Delay and Collision Reduction Mechanism for Distributed Fair Scheduling in WIreless LANs", ICCSA, May, 2004. 